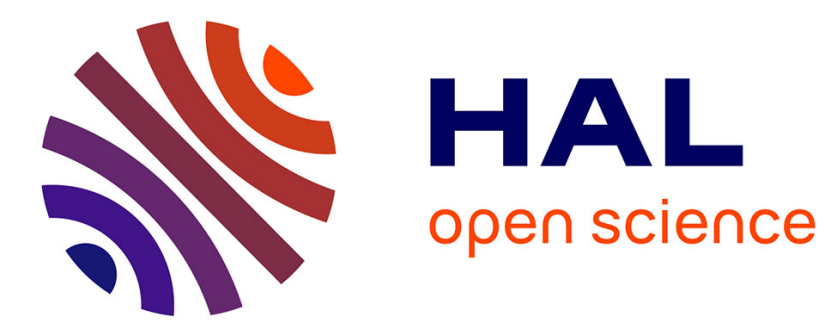

\title{
Quantum mechanics for space applications
}

Alexandre Bresson, Yannick Bidel, Philippe Bouyer, Bruno Leone, Eamon

Murphy, P. Silvestrin

\section{To cite this version:}

Alexandre Bresson, Yannick Bidel, Philippe Bouyer, Bruno Leone, Eamon Murphy, et al.. Quantum mechanics for space applications. Applied Physics B - Laser and Optics, 2006, 84, pp.545-550. 10.1007/s00340-006-2412-x . hal-00106374

\section{HAL Id: hal-00106374 https://hal.science/hal-00106374}

Submitted on 15 Oct 2006

HAL is a multi-disciplinary open access archive for the deposit and dissemination of scientific research documents, whether they are published or not. The documents may come from teaching and research institutions in France or abroad, or from public or private research centers.
L'archive ouverte pluridisciplinaire HAL, est destinée au dépôt et à la diffusion de documents scientifiques de niveau recherche, publiés ou non, émanant des établissements d'enseignement et de recherche français ou étrangers, des laboratoires publics ou privés. 
App. Phys. B manuscript No.

(will be inserted by the editor)

\section{Quantum Mechanics for Space Applications ${ }^{\star}$}

\author{
A. Bresson ${ }^{1}$, Y. Bidel ${ }^{1}$, P. Bouyer ${ }^{2}$, B. Leone $^{3}$, \\ E. Murphy ${ }^{3}$, P. Silvestrin ${ }^{3}$ \\ 1 ONERA : Office National dEtude et de Recherches Arospa- \\ tiales \\ Chemin de la Hunire, 91761 Palaiseau, France \\ 2 Laboratoire Charles Fabry de IInstitut dOptique \\ Centre National de la Recherche Scientifique et Universit \\ Paris Sud 11 \\ Bt. 503, Campus Universitaire dOrsay, 91403 Orsay Cedex, \\ France \\ 3 ESA/ESTEC, European Space Agency \\ European Space Research and Technology Centre \\ Keplerlaan 1, 2201 AZ Noordwijk ZH, The Netherlands
}

The date of receipt and acceptance will be inserted by the editor

\begin{abstract}
This paper is an introduction to the following articles in the scope of the Quantum Mechanic for Space Study initiated by ESA and lead by ONERA. The context of Quantum Mechanics for Space is summarised, and fields under development concerned are briefly introduced. On one hand, technological applications in space of quantum mechanics are explored and on the other hand some test of quantum mechanics are outlined. We also give a brief presentation of the Opto-electronics Section at the European Space Agency and on the technology development activities it carries out, with particular emphasis on those activities related to the topics of interest of the Quantum Mechanics in Space workshop. As an example, a summary of two ESA studies on gravity gradiometry and their relevance to the field of atomic interferometry is given. In the light of the scientific requirements, derived for both Earth Observation and Planetology for future space missions, atom interferometry shows promise and may be at an advantage with respect to currently available accelerometer and inertial sensor systems.
\end{abstract}

\section{Quantum Mechanics and space}

Quantum mechanics (QM) is a theory that had been developed at the beginning of the previous century. One of

\footnotetext{
^ http://qm-space.onera.fr
}

the main idea in this theory is the wave particle duality : particles, for example electrons, can behave like waves and waves, for example light, can be seen like particles. QM was able to explain many phenomena - ranging from the black body radiation to atomic spectra - and is now a well establish theory that described the behaviour of the matter at the microscopic scale. However, some aspects of QM theory remain unsolved - like the problem of the measurement or the unification with general relativity - or some phenomena are not yet fully understood - like for example the superconductivity - and there is thus still an important research effort in QM both theoretically and experimentally. Although rather new and fundamental, QM already extends its interest beyond the academic research and has already lead to a wide range of technologies like the laser, the transistor or the atomic clocks. Nowadays, there is even a boost in QM based technologies - for example with quantum information.

Many of these technologies - like quantum detector or atomic clocks - may find their ultimate use and performances when applied in space mission since space and in particular microgravity can provide a very attractive environment. In space, the microgravity allows to obtain long interaction time and therefore to make extremely precise measurement, and it also provides a quiet and isolated environment where a better understanding of QM can be acheived - such as for example a study of quantum decoherence. QM and space have thus an 'intimate' relationship. One can use space to study QM and one can use QM based technology for space mission. In order to identify the most promising field of QM in space, ESA initiated the QM for space study. This project has been lead by ONERA and was made with the co-operation of famous research institute or laboratories: IOTA (Orsay, France), SYRTE (Paris, France), IQO (Hanover, Germany), and University of Strathclyde(UK). A roundtable has been organised in the frame of the project. This event has outlined the developments in QM based technologies for space applications and tests by bringing together European experts in the relevant theoretical and technological domains. It has been the opportunity to build together new ideas and perspectives for applications of QM, and to establish a link between ESA and this scientific community. Based on several criteria like the needs resulting from space missions, the scientific maturity or the global interest, the $Q M$ for space study selected five relevant fields :

\subsection{Clocks}

Atomic clocks are the best-known QM atomic interferometer and realise the best level of stability any accuracy of any kind of measurement. Space can improve the accuracy and the stability of this clock by providing longer interaction time. PHARAO a project of atomic 
space clock is under development. Atomic clocks can be used to test quantum mechanics like the possible variation of the fine structure constant. Atomic clocks can also be used to improve the precision of some device like position system.

\subsection{Atomic inertial sensor}

Atomic Quantum Sensors have a large potential to become a key-technology for the ultra-precise monitoring of accelerations and rotations. These sensors evolved out of a new kind of optics based on matter waves rather than light waves. Matter wave optics is still a young, but rapidly progressing science. Space, by providing longer interaction time, can improve the precision of these sensors. Atomic inertial sensors in space can be used to test general relativity and quantum mechanics. They can also be used in geophysics to map the Earth geopotential or in inertial navigation to determine precisely the position, velocity of a satellite.

\subsection{Low temperature and condensed matter: SQUID}

The SQUID (Superconducting Quantum Interference Devices) is a detector of magnetic flux with an energy sensitivity unequalled by any other device. It can be configured to measure many physical quantities, with an electromagnetic energy resolution that can approach the quantum limit.

\subsection{BEC, Atom Laser and Macroscopic Quantum Matter}

Bose Einstein condensate is a state of the matter where a macroscopic fraction of the atoms are in the same quantum state. It has been obtained for the first time in 1995. Nowadays, Bose Einstein condensates are used in many experiments as a model system to study many body physics like superfluidity or quantum transition. Doing experiment of Bose Eintein condensate in microgravity can allow us to obtain lower temperature and study BEC transition with unprecedented detail. We can also address the fundamental question of coherence in a space born BEC in a free falling environment that is perfectly isolated from external influence.

\subsection{Quantum detectors: QWIP}

QWIP stands for Quantum Well Infrared Photodetector. QWIP is an advanced nanodevice that is fabricated using state-of-the-art semiconductor technology. The flexibility of the precision QWIP design and fabrication technology allows it to be optimised for a variety of mission conditions. Based on highly mature GaAs growth and processing technology, QWIPs can be used to produce large-format focal plane arrays for high-resolution infrared cameras at lower cost than any other competing technologies. The manufacturability of QWIP-based advanced infrared camera has enabled a rapid commercialisation of this technology. The availability of QWIP cameras has already contributed to the advancement in medical applications. A novel QWIP-based breast cancer detection technology was recently developed in the US. The high operability, high uniformity, high stability, and high radiation tolerance of QWIP cameras remarkably simplify the on-board and ground based data analysis for space missions, thus providing a significant cost reduction in software development, data processing and major system level benefits upon integration. As such, the QWIP technology will enable a vision of frequent and low cost missions in the 21st century.

\section{Outcome of the $Q M$ for space study}

The outcome of this study was that QM might needs space to be ultimately tested and that space mission will benefit from QM base technologies. If these technologies reach maturity fast enough, many space missions, already studied or to come, will use it. This maturity can be reached if an important development work for some crucial key elements:

- Laser sources (integration of laser beam generation): lots of technology (and experiments) are based on the utilisation of laser. Today, the lack of efficient spatial version concerning laser is the major limitation.

- Atom sources: as laser sources for optical interferometer, spatial atomic sources will be needed to permit the use of (cold and degenerate) atoms as a quantum source for various and promising applications.

- Low temperature devices: using low temperature implies a large effort concerning the development of solid state refrigeration suitable for space environment. For example, it is expected that this development will replace liquid cryogen tanks for future cryogenic and ultralow temperature space missions.

- Volume / Mass reduction

- Maturity improvement on ground experiments: today, all quantum technologies and tests experiments are on the edge of knowledge. Ground experiments will be always needed for the preparation of space quantum mission and for the improvement of scientific quantum knowledge.

\section{Opto-electronics Section Activities at ESA (TEC-MME) and QM for space}

\subsection{Introduction}

The Opto-electronics Section, better known within ESA by its address code, TEC-MME, belongs to the Mecha- 
tronics and Optics Division (TEC-MM) within the Mechanical Engineering Department (TEC-M) of the Technical and Quality Management Directorate (D/TEC). As the name suggests, this directorate deals with the management of technical and quality aspects of the agency work including technology development and space qualification activities. Hence, the Opto-electronics Section deals with all opto-electronics technology development activities at ESA.

\subsection{TEC-MME Terms of Reference}

The term "opto-electronics" may encompass more or less technical domains according to the context it is being used in. In order to avoid confusion, the specific competences of the Section are defined in the TEC-MME terms of reference. These are listed below:

- Opto-electronic device technologies and applications, including photonic integrated optics;

- Solid state lasers;

- Semiconductor laser diode technology;

- Non-linear optics;

- Superconductor technology;

- Far infrared heterodyne instrument design and verification above $1 \mathrm{THz}$;

- Detector technology and radiometry for large portions of the electromagnetic spectrum, namely X-ray, UV, Visible, IR, Far-IR above $1 \mathrm{THz}$.

This broad range of competences translates into several tens of technology development activities and studies managed by around ten staff led by the Head of Section, Errico Armandillo.

\subsection{Quantum Mechanics in Space Activities}

Let us now focus on those activities carried out by TECMME relevant to the topic of the present workshop. Quantum mechanics in space is a very broad subject and may encompass too large a number of topics to be covered by a single workshop. Fortunately, the workshop organizers were able to identify the most relevant ones, namely:Atomic Clocks; Low-Temperature Devices; Fundamental Physics in Space; Quantum Decoherence; Quantum Well Detectors; Quantum Cascade Lasers; Spintronic; Atomic Interferometers; BEC QED; Quantum Gravity; $Q M$ for Space $\mathcal{G}$ Earth $\mathrm{Ob}$ servation ${ }^{1}$.

3.3.1 Laser pumped Atomic Clocks Technology development activities in the area of atomic clocks have been going on for some time within TEC-MME and continue to do so. An activity on laser-pumped Rubidium gas cell

\footnotetext{
1 The ones that are cover by TEC-MME are highlighted in italics.
}

standards has been completed recently while another on laser-pumped Caesium beam clocks is ongoing.

Two new activities, one on gas cell laser clock technologies and another on a feasibility study of laser-pumped Caesium clock for Galileo are about to begin. Finally, an activity on miniature laser pumped atomic clocks and another one on Optical frequency Standards (Ytterbium, Strontium, Mercury, Calcium) have been proposed and are required for a plethora of future space applications. The development work on the Optical Frequency standards would provide one of the key developments required for the realisation of an Optical Atomic Clock.

Finally, the commitment of TEC-MME in the domain of atomic clocks and related technologies is especially evident in the effort and far-sightedness demonstrated by the setting up of the "1st ESA international workshop on Optical Clocks" on 8 - 10 June 2005 at ESTEC.

\subsubsection{Laser-cooled Atom Interferometry The field of laser-} cooled atom interferometry is still relatively new at ESA. Nevertheless, the European Space Agency has already been quite involved in this domain thanks to the HYPER mission, which was the subject of both an internal Concurrent Design Facility study at ESTEC and an industrial study led by Astrium GmbH. The industrial study led to a technology development roadmap for, amongst other things, atom interferometry. Currently, only one of these recommended technology development activities for atom interferometry is being pursued at ESA. This activity is entitled "Laser-cooled Atom Sensor for UltraHigh-Accuracy Gravitational Acceleration and Rotation Measurements" and deals with the optimisation of an atom source for space application and is led by TECMME.

\subsubsection{Quantum Opto-Electronics}

Quantum Well Detectors: The specifications for the Darwin mission linear detector array are very demanding both in terms of wavelength band, noise performance and operating temperature. Presently, an extrinsic semiconductor technology developed for JWST is well established and satisfies the performance requirement albeit at an operating temperature lower than would be desirable. We are currently running two activities - Far IR Linear Detector Array and QWIP Optimisation for Darwin FIR Linear Array - to develop Quantum Well Infrared Photoconductor (QWIP) technology aiming at increasing the operating temperature and optimise the detectors for different wavelength bands. State-of-theart results in the field have already been obtained, although the indication is that the required level of dark current will be at least one order of magnitude above the required Darwin specifications. However, these results could be of interest to Earth Observation and an 
activity is ongoing to develop this technology for thermal imaging with a different set of requirements.

Quantum Cascade Lasers : In the field of heterodyne detection, far infrared sources are required to act as local oscillators for THz mixers. Within this framework, an activity on Optical Far-IR Wave Generation was successfully completed resulting in the realisation of a Quantum Cascade Laser device demonstrator operating at $3 \mathrm{THz}$.

3.3.4 Low Temperature and Superconducting Devices Low temperature devices development has a substantial heritage at TEC-MME. In particular, superconductor-based detectors have been or are being investigated and developed as detectors for $\mathrm{THz}$ waves, X-rays and gravity gradients. TEC-MME pioneered the development of superconducting $\mathrm{THz}$ heterodyne mixers at ESA in the form of Superconductor-Insulator-Superconductor and Hot Electron Bolometer mixers. Both types of mixers have been incorporated in actual $\mathrm{THz}$ mixer block design of the HIFI instrument on the Herschel Space Observatory scheduled for launch in 2007. TEC-MME is also currently supporting the Space Science Directorate in the investigation of superconductor Transition Edge Sensors for X-ray detection. In particular, an activity is currently ongoing to study the device physics and noise analysis of these X-ray calorimeters. Superconductor-based sensors have also been investigated in the context of the next generation accelerometers and inertial sensors for post-GOCE gravity gradiometry missions. The results of the study, however, pointed to atom interferometry as a more promising technology for future applications in this field. Finally, TEC-MME has been involved in the Biannual WOLTE Conference on Low Temperature Electronics since its inception about a decade ago and has been hosting this workshop twice. This year the workshop is againe organised by TEC-MME: "Seventh International Workshop on Low Temperature Electronics", WOLTE-7, 21 - 23 June 2006, ESTEC.

\subsection{Support to Earth 6 Space Sciences}

As was explained in the opening paragraph, the Optoelectronic Section ultimately belongs to the Technical and Quality Management Directorate. As such its main mission is to technically support the project directories, such as the Earth Observation and Science Directories, in the preparation and realisation of space missions. In this capacity, TEC-MME has been offering continuous support to a number of studies and running projects relevant to the topics of the current workshop. In particular, support has been given to the Earth Observation study "Enabling Observation Techniques for Future Solid Earth Missions" and a study on "Gravity Gradient Sensor Technology for Planetary Missions" is being performed to support Planetary Sciences at ESA. Both studies suggest that a major improvement in accelerometer and inertial sensor sensitivity will be required of follow-on gravity gradiometry missions, both for Earth Observation and Planetology. At the time of writing such sensitivity may only be achieved by atomic interferometry methods, at least in principle. In addition, the setting up of the First ESA International Conference on Optical Clocks is preparing the terrain for a number of applications that may have implications to future Space and Earth Sciences as well as Navigation missions. In the future, one may envisage TEC-MME involvement in the topics of Ultra-sensitive Gravimetry - using very precise atomic clock, Atom Interferometry and the development and space qualification of Optical Clock to check for variations in Fundamental constants

\subsection{Conclusion}

The Opto-electronics Section ant ESA possesses the competence and is committed to a large cross section of topics and activities relevant to this workshop. Therefore, it is a point of contact at ESA for the community of scientists represented at this workshop who are planning to develop their technology for space applications. In particular, for future ESA missions incorporating matterwaves or ultra-precise metrology concepts, the development of the following technologies will be needed:

- Atom Optics: space qualified stable Source of Cold Atoms

- Compact laser sources for cold atom production: to cool down atoms and control atomic beams

- Ultra-stable Raman Lasers: for coherent matter wave splitting

- Optical frequency synthesizer: space qualifiable femtosecond comb

- Realisation of a feasible Optical Frequency standard/s for space: select most suitable option from the choices available

- Realise a completely optical atomic clock: design and verification.

TEC-MME is looking forward to living up to the challenge.

\section{Atom Interferometry Needs for Future Gravity Gradiometry Space Missions}

\subsection{Introduction}

Two studies on future gravity gradiometry missions have or are being performed at ESA. One of them has recently been completed. It builds on the data accumulated over the past missions dedicated to the measurement of the Earth's gravity field to elaborate on the scientific and technological needs of future gravity geopotential missions. The other study reviews the existing gravity field 
data of a number of scientifically relevant moons and planets in our solar system in order to derive the scientific and engineering requirements for a compact gravity gradiometer payload on board a planetary satellite mission.

\subsection{Geopotential Missions}

The gravity field does not have the same value at all points on the Earth's surface. This is due to the fact that the Earth is neither perfectly spherical nor homogeneous. The deviation from the spherical shape is partly due to the Earth's rotation, which flattens it at the poles making it approximate the shape of an ellipsoid, while the heterogeneous density of the Earth is responsible for deviations in both sphericity and gravity field value.

The geoid is an equipotential surface of the Earth's gravity field. To visualise the geoid one may imagine the Earth as being completely covered in non-circulating water. In such a hypothetical case, the shape of the Earth would tally with the geoid. Deviations of the geoid from the theoretical rotation ellipsoid are called geoid anomalies and are up to a few metres. Similarly, deviations of the actual gravity field from the theoretical value of the gravity field normal to the surface of the rotation ellipsoid are called gravity anomalies. These are measured in $\mathrm{mGal}^{2}$. A geopotential space mission uses satellites to map the global gravity field. This can be achieved by measuring the geopotential second order derivatives, i.e. the (relative) accelerations of proof masses, at the satellite(s)'s orbit. The second order derivatives are then used in an inverse problem method to calculate the spherical harmonic expansion coefficients of the geopotential. To every harmonic degree $l$ of the expansion corresponds a spatial wavelength equal to the Earth circumference divided $l(\lambda=40.000 \mathrm{~km} / l)$. Therefore the harmonic degree defines the spatial resolution down to which the geoid may be known.

The geopotential itself is not a simple entity. It is the lumped sum of the gravitational effects of all the phenomena occurring both on the surface of the Earth and in its interior. These include all solid, liquid and gaseous components contributing to the gravity field, such as: Solid Earth, Volcanic activity, Water cycles, Oceans, Ice sheets, Glaciers, Sea level, Atmosphere.

It is important to note that, traditionally, these components have been studied separately giving rise to several disciplines including, amongst others: Solid Earth, Geophysics, Volcanology, Hydrology, Oceanography and Meteorology.

As a result, the knowledge base to be used for the preparation of a geopotential mission is very broad and has often ill-defined interfaces. These may sometimes represent geographical mismatches within a single discipline. In fact, what makes a space-based geopotential

\footnotetext{
${ }^{2} 1 \mathrm{Gal}=1 \mathrm{~cm} / \mathrm{s}^{2}, 1 \mathrm{mGal} \approx 10^{-6} \mathrm{~g}$
}

mission so attractive is the ability to provide a global picture both geographically and interdisciplinary, thereby contributing to the gradual creation of a unified model for the Earth system.

Still, the lumped signal that is obtained from the mission must be supported by both ground data and modelling in order to be able to separate the different contributions and avoid as much as possible aliasing of the different signal sources.

Therefore, in order to minimise these problems it is desirable to obtain as complete a geopotential map as possible. One is usually interested in maximising both spatial resolution and mission lifetime, the latter in order to improve accuracy and also to estimate gravity field changes. Unfortunately, these two requirements work against each other. On the one hand, in order to increase spatial resolution one must decrease the satellite(s)'s altitude as much as possible since the gravitational signal decreases with the square of the radius from the gravitating body's centre. On the other hand, in the presence of an atmosphere, a low altitude requires the consumption of large quantities of propellant to compensate for air drag. This in turn severely limits the lifetime of the mission. In addition, the temporal resolution is also an important parameter in the equation and depends, amongst other things, on the orbital parameters.

\subsection{Missions Types}

Different types of baseline mission configurations for past present and future geopotential missions exist. These will be briefly reviewed in this section.

4.3.1 High Earth Orbit The High Earth Orbit (HEO) mission configuration is the first type ever to be used as a geopotential mission. The LAGEOS[1] missions represent an example of this configuration. They consist of a passive reflector payload on a satellite at high altitude. The satellite is very precisely laser-tracked from reference ground stations and the analysis of orbital data provides the gravity field information.

The high altitude serves two purposes. Firstly, the effect of the non-gravitational force is very much reduced by the almost total lack of atmospheric drag and by selecting an appropriate aerodynamic design. The remaining non-gravitational forces, such as solar pressure, are removed by modelling. Secondly, the high altitude also acts as a natural band-pass filter for low degree spherical harmonics that can be used to isolate processes associated with the Earth's interior.

In this configuration the proof mass is the satellite itself and the fact that its accelerations cannot be monitored continuously and in three dimensions constitutes a drawback. However, this configuration is certainly the simplest to implement. Furthermore, this type of mission has a very long lifetime because it does not require 
an active payload, such as a drag-compensating Attitude and Orbit Control System (AOCS).

4.3.2 High-Low Satellite-to-Satellite Tracking The HighLow Satellite-to-Satellite Tracking (HLSST) configuration consists of a Low Earth Orbit (LEO) satellite tracked by a HEO GNSS constellation as is done for CHAMP[2]. In this configuration the non-gravitational forces need to be measured by accelerometers in order to correct for them either by the use of drag-compensating AOCS or by later correction of the raw data. The advantage of this configuration is the access to higher harmonics and therefore higher spatial resolution information than in the HEO case. The limitation lies in the tracking accuracy of the GPS constellation used.

\subsection{Low-Low Satellite-to-Satellite Tracking}

While the two previous mission configurations relied on the external tracking of a proof mass the Low-Low Satelliteto-Satellite (LLSST) is based on the measurement of the relative acceleration of two or more proof masses. The measurement is achieved by inter-satellite ranging as is done for GRACE[3]. HLSST is also realised in GRACE.

In the case of GRACE, continuous microwave tracking is the ranging method of choice. However, for future missions laser ranging is also envisaged. The ranging measurement provides differential metrological information. This must be combined with GPS tracking to obtain absolute metrology and position information of the satellites. Again, because of the LEO, accelerometers are needed to monitor and/or compensate for nongravitational forces.

\subsubsection{Satellite Gravity Gradiometry In the Satellite} Gravity Gradiometry (SGG) configuration the proof masses are placed within the satellite and their differential accelerations are monitored. In other words, the mission payload consists of sensitive accelerometers, which are also used to monitor or compensate the non-gravitational forces. Such a mission configuration is represented by GOCE[4] - Gravity Field and Steady-State Ocean Circulation Explorer. More details on this mission will be provided in a later section.

Of the four configurations summarised above, two of them, HEO and SGG, have been identified as being particularly relevant to the topic of this workshop. These will be discussed in turn in the following sections.

\subsection{HEO Mission}

The ESA study entitled "Enabling Observation Techniques for Future Solid Earth Missions" [5] identified an improvement to the HEO configuration. GNSS-tracking and accelerometers in addition to laser tracking from ground would enable continuous three-dimensional tracking for improved low harmonic Solid Earth applications. Furthermore, the presence of a high precision $\left(10^{-16}\right)$ atomic clock on board the satellite would provide the possibility to interweave gravity field measurement and positioning. Such a mission could be of interest for Earth sciences, time keeping, fundamental physics and, possibly, telecommunication. However, because of the presence of high precision active components the mission lifetime would be considerably shorter than for a LAGEOStype mission.

\subsection{Future needs for $S G G$ Missions}

Another promising mission configuration identified by the study and of interest to the present workshop concerns the SGG type mission. One such mission is ESA's GOCE - Gravity Field and Steady-State Ocean Circulation Explorer - due for launch in 2006. This is a satellite in sun-synchronous orbit at $240 \mathrm{~km}$ altitude. The main payload is a three-axis gradiometer capable of measuring the diagonal gravity gradient tensor components. The predicted accuracy of the gradiometer is, depending on the axis, 100 to $6 \mathrm{mE} / \sqrt{\mathrm{Hz}}$ between 100 and $5 \mathrm{mHz}^{3}$.

Clearly, any follow-on mission to GOCE will have to perform even better given these already quite challenging figures. Indeed, for such a mission to make sense scientifically, the study quoted required sensitivities down to $0.1 \mathrm{mE} / \sqrt{\mathrm{Hz}}$ between 100 and $0.1 \mathrm{mHz}$. In addition, pointing rate knowledge of $4 \times 10^{-11} \mathrm{rad} \mathrm{s}^{-1} / \sqrt{\mathrm{Hz}}$ will also be needed. It is currently believed that these figures cannot be obtained with presently available three-axis gradiometers concepts. A new gradiometer technology is needed. This is a challenge that the Atom Interferometry scientific community may wish to take up.

\subsection{Planets and Moons}

Though understanding planet Earth as a system is vital for the survival of the human race, our understanding of other planetary systems may also contribute to a deeper knowledge of the processes that occur here on Earth. The planets and moons that may be best observed are located within our solar system and, so far, constitute the main object of Planetology. These planets and moons may be regarded as experimental test beds of what our planet might have looked like under different conditions or at different points in time. Therefore, it is of utmost importance to increase our knowledge of the structure, both internal and external, of these celestial bodies. In particular, the interior structure may be known through their gravity field.

So far, planetary gravity filed knowledge has been inferred from Line-of-Sight (LoS) observations, obtained

\footnotetext{
${ }^{3} 1 \mathrm{E}=10^{-9} \mathrm{~s}^{-2}$
} 
by analysing the radio signal from a satellite in orbit around a planet or moon.

\subsection{Future Needs for Planetology}

The second ESA study entitled "Gravity Gradient Sensor Technology for Future Planetary Missions" [6] formulated the scientific requirements for a gravity gradiometer payload on board a still hypothetical planetary mission. The study focused on the most scientifically relevant planets and moons, such as Mars, Europa, Titan and Mercury. The preliminary requirement for the gradiometer sensitivity is $1 \mathrm{mE} / \sqrt{\mathrm{Hz}}$ between 10000 and 1 $\mathrm{mHz}$. The reason that this requirement is so challenging is due to the good quality of the existing LoS results. This poses a problem for the technology to be used for the payload since engineering requirements are very much constrained in size, mass and power consumption as all planetary missions are. A technology review was carried out and considered various technologies, including: superconducting devices, Micro Electro-Mechanical Systems (MEMS) and Laser-Cooled Atoms.

Atom Interferometry (AI) was selected as promising because impressive differential acceleration sensitivities have been demonstrated on the ground and a significant improvement can be extrapolated for operation in space. Indeed, sensitivity increases with the square of the interrogation time, which is limited on the ground to a few hundred milliseconds due to gravity. Microgravity allows for much longer interrogation times. For instance, one can extrapolate a three-orders-of-magnitude improvement on sensitivity for an interrogation time of 10 seconds. In contrast, other technologies considered during the study may show varying degrees of promise but major technological hurdles still need to be overcome before the required sensitivity is achieved on the ground. In this respect, AI may soon become the necessary technology push for the realisation of a planetary gravity gradiometer. However, a major miniaturisation effort remains to be done to satisfy the compactness required for a planetary mission.

The different frequencies of laser beams for trapping, detection, Raman pulses are provided by two lasers and acoustic-optic modulators. The laser beams are transported using optical fibres and are shared for both measurements of gravity $g_{1}$ and $g_{2}$. A very high common mode noise rejection can be achieved by using the same Raman beam for a simultaneous measurement. This concept assumes a miniaturisation of both cooling and interrogation laser systems and optics. Furthermore, it assumes the following technical parameters: Vacuum chamber size of $\sim 10 \mathrm{~cm}$; Atom cloud size of $\sim 5 \mathrm{~mm}$; Atomic species of Cs or Rb; Baseline of $1 \mathrm{~m}$; Interrogation time of $10 \mathrm{~s}$; Weight of a few $\mathrm{kg}$. With these values the gradiometer could in principle achieve a sensitivity of $1 \mathrm{mE} / \sqrt{\mathrm{Hz}}$ at $0.1 \mathrm{~Hz}$.

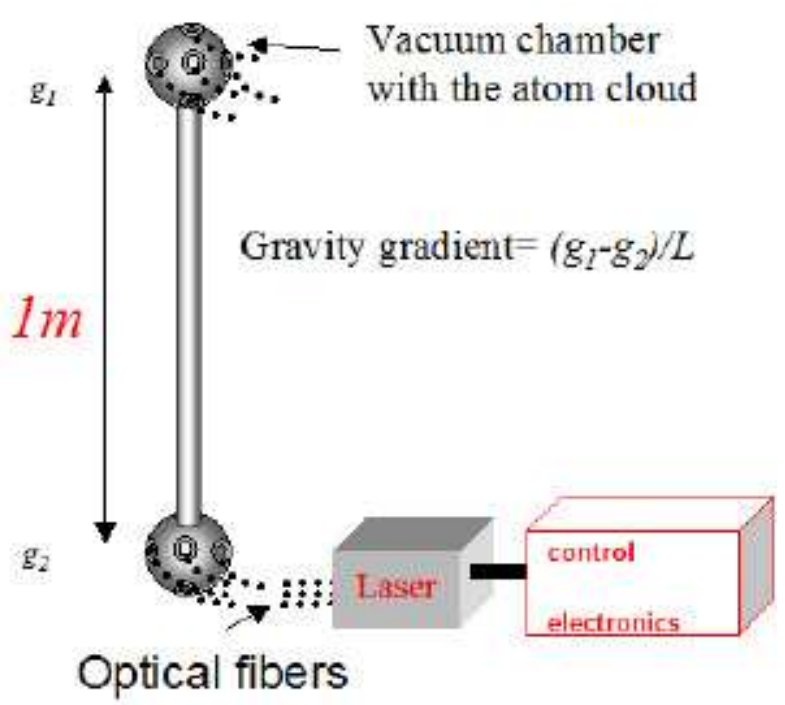

Fig. 1 Back of the envelope design provided in the study. The gravity gradient is deduced by measuring gravity at two points, g1 and g2, separated by the baseline L.

\section{References}

1. http://www.earth.nasa.gov/history/lageos/lageos.html

2. http://www.gfz-potsdam.de/pb1/op/champ/index_CHAMP.html

3. http://www.gfz-potsdam.de/pb1/op/grace/index_GRACE.html

4. http://www.esa.int/esaLP/LPgoce.html

5. R. Rummel, J. Flury et al., ESA Study: "Enabling Observation Techniques for Future Solid Earth Missions", Technical Note 1: "Science Objectives for Future Geopotential Field Mission", Doc. Ref. SOLIDEARTHTN-TUM-001, 1 November 2003.

6. J. Flokstra, R. Wiegerink, H. Hemmes, J. Sesé, ESA Study: "Gravity Gradient Sensing Technology for Future Planetary Missions", Technical Note 3: "Gravity gradient sensor concepts and related technologies for planetary/lunar missions", Doc. Ref. ESA ITT A0/13829/01/NL/ND WP3000, December 2004. 